\title{
Sgr A West in the light of molecules: cold and dense gas east of the circumnuclear disk
}

\author{
${\text { Lydia } \text { Moser }^{1} \text {, A. Eckart }}^{1,2}$, A. Borkar ${ }^{1,2}$, M. García-Marin ${ }^{1}$, \\ D. Kunneriath ${ }^{3}$, B. Jalali ${ }^{1}$, N. Sabha ${ }^{1,2}$, B. Shahzamanian ${ }^{1,2}$,
} M. Valencia-S. ${ }^{1}$, M. Zamaninasab ${ }^{2}$, L. Bronfman ${ }^{4}$ and R. Finger ${ }^{4}$

${ }^{1}$ Physikalisches Institut, University of Cologne, Zülpicherstr. 77, 50937 Cologne, Germany email: moser@ph1.uni-koeln.de

${ }^{2}$ Max-Planck-Institut für Radioastronomie, Auf dem Hügel 69, 53121 Bonn, Germany

${ }^{3}$ Astronomical Institute of the Academy of Sciences, Bocni II 1401, CZ-141 00 Praha 4 Sporilov, Czech Republic

${ }^{4}$ Departemento de Astronomia, Universidad de Chile, Castilla 36-D, Santiago, Chile

\begin{abstract}
We present the very first detection of $\mathrm{N}_{2} \mathrm{H}^{+} J=(1-0)$ and $\mathrm{CH}_{3} \mathrm{OH}\left(2_{k}-1_{k}\right)$ line emission on $5^{\prime \prime}$ scales in the circumnuclear disk (CND) around Sgr A*. The emission matches the position and shape of the dark clouds in the near-infrared. Our findings suggest that these molecular clouds in the eastern CND are significantly colder and denser than the rest of the CND, and partially shocked. The research on these dark clouds will contribute to understanding the processes of star formation close to a supermassive black hole.
\end{abstract}

Keywords. molecular data, ISM: clouds, ISM: molecules, Galaxy: center, Galaxy: nucleus

\section{Introduction}

The Galactic center is a unique laboratory to investigate the complex physical processes taking place in the vicinity of a supermassive black hole ( $\left.\mathrm{Sgr} \mathrm{A}^{*}\right)$, i.e. the matter transport to the center and star formation in such a violent environment. We have detected $\mathrm{N}_{2} \mathrm{H}^{+}$ $J=(1-0)$ and $\mathrm{CH}_{3} \mathrm{OH}\left(2_{k}-1_{k}\right)(96 \mathrm{GHz})$ line emission in the circumnuclear disk (CND). The observations have been conducted with the Combined Array for Research in Millimeter-wave Astronomy (CARMA) in continuum mode around $95 \mathrm{GHz}$. The data comprise also the emission of $\mathrm{H}^{13} \mathrm{CO}^{+} J=(1-0)$, $\mathrm{SiO} J=(2-1)$, $\mathrm{HCN} J=(1-0)$ and $\mathrm{HNC} J=(1-0)$.

\section{Results}

The emission of $\mathrm{N}_{2} \mathrm{H}^{+}$and $\mathrm{CH}_{3} \mathrm{OH}$ mimics the distribution of the $\mathrm{H}_{2} \mathrm{CO}$ emission (Martín et al. 2012) and of the dark clouds in the near-infrared (Figure 1): It is strong in the northeast arm (A, B) and the region (C) east of the southern extension (nomenclature as in Martín et al. 2012) and faint in the central ring outlined by the SiO. In cold, dark clouds, the $\mathrm{N}_{2} \mathrm{H}^{+}$abundance is high when its main destroyer $\mathrm{CO}$ is depleted by freezeout onto dust grains ( $\mathrm{T}=20$ - $25 \mathrm{~K}$, Vasyunina et al. 2011, and references therein). Indeed, the $\mathrm{N}_{2} \mathrm{H}^{+} / \mathrm{H}^{13} \mathrm{CO}^{+}$line ratio in $\mathrm{A}, \mathrm{B}$ and $\mathrm{C}$ is about 5 , which is exceptionally high compared to the rest of the CND $(<1)$, indicating the presence of cold, dense gas (Sanhueza et al. 2012). This is further supported by a $\mathrm{HNC} / \mathrm{HCN}$ line ratio $>0.3$ in these regions, which is higher than that of the CND $(<0.2)$ : HNC may be preferentially formed in cold environments $(\mathrm{T}<24 \mathrm{~K}$, Hirota et al. 1998). 

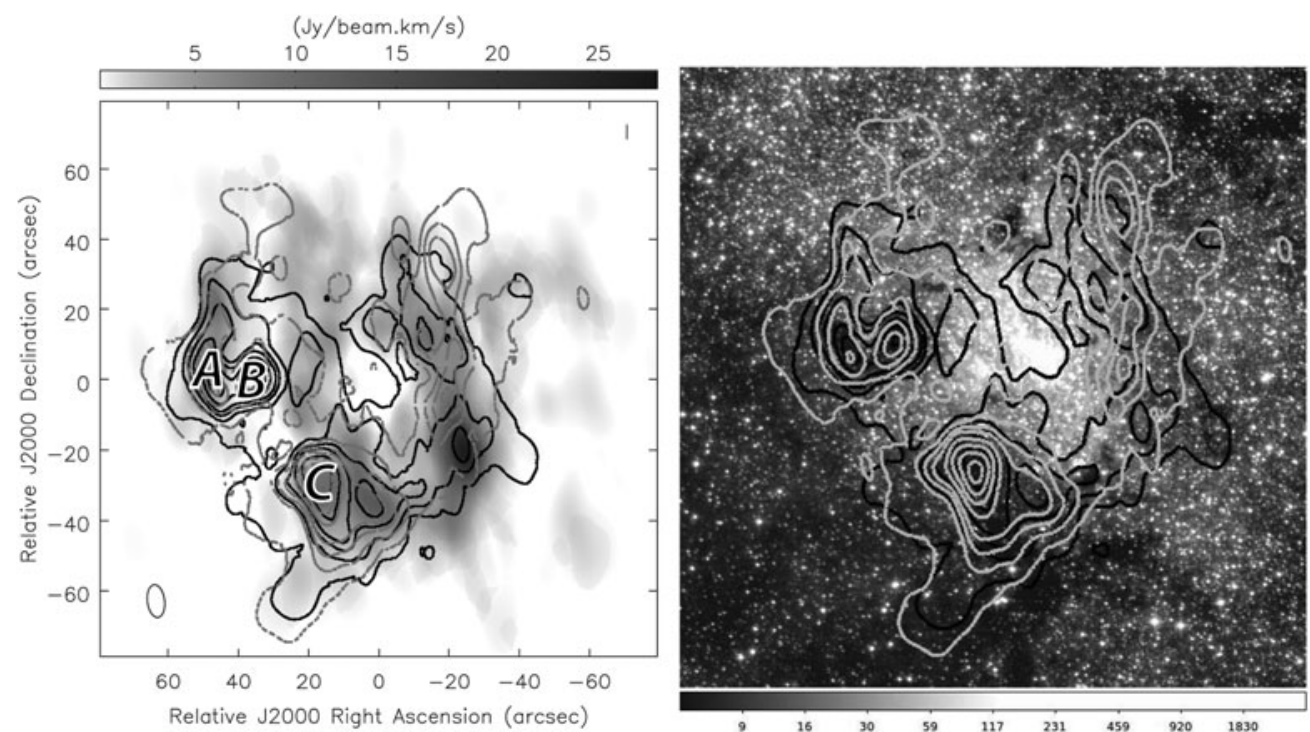

Figure 1. $\mathrm{N}_{2} \mathrm{H}^{+}$(black) and $\mathrm{CH}_{3} \mathrm{OH}$ (grey dashed) in contours of 3, 6, 9, 12, 18, 24, 30 (, 36 for $\left.\mathrm{CH}_{3} \mathrm{OH}\right)$ Jy beam ${ }^{-1} \mathrm{~km} \mathrm{~s}^{-1}$ with a beam size of $9.5^{\prime \prime} \times 5.0^{\prime \prime}$. Both are overlayed on our $\mathrm{SiO} J=(2-1)$ map in greyscale (left) and on an Hubble NICMOS $1.87 \mu \mathrm{m}$ image (right, MAST/STScI - GC Pa $\alpha$ survey).

$\mathrm{H}_{2} \mathrm{CO}$ and $\mathrm{CH}_{3} \mathrm{OH}$ are efficiently formed on dust grains (Shalabiea \& Greenberg 1994, Watanabe \& Kouchi 2002), which explains the coincidence of their distribution. The presence of these species in the the vicinity of the strong UV radiation from the nuclear stellar cluster (Martín et al. 2012, Yusef-Zadeh et al. 2013, and references therein) suggests self-shielding of the clumps. $\mathrm{N}_{2} \mathrm{H}^{+} / \mathrm{CH}_{3} \mathrm{OH}$ line ratios in clumps $\mathrm{A}$ and $\mathrm{B}$ are higher $(\sim 2.5)$ than in $\mathrm{C}(\sim 0.8)$ indicating different conditions. Shocks are the best explanation for this increased release into the gas-phase: the $\mathrm{CH}_{3} \mathrm{OH}$ (and $\mathrm{H}_{2} \mathrm{CO}$ ) emitting regions $\mathrm{A}$ and $\mathrm{C}$ are also traced by $\mathrm{SiO}$ (Figure 1). The likely origin of the shocks is the expanding shell of Sgr A East interacting with the $50 \mathrm{~km} \mathrm{~s}^{-1}$ GMC and compressing the gas (e.g., Martín et al. 2012). This is supported by the clumps' velocities of $\sim 50$ $\mathrm{km} \mathrm{s}^{-1}$. A detailed discussion of the full data set will be published in Moser et al. (2014; in prep.).

\section{Conclusions}

We have obtained unprecedented maps of $\mathrm{N}_{2} \mathrm{H}^{+}$and $\mathrm{CH}_{3} \mathrm{OH}$ emission in the CND. We suggest that the molecular gas in the northeast $\operatorname{arm}(\mathrm{A}, \mathrm{B})$ and the region $(\mathrm{C})$ east of the southern extension is significantly colder and denser than the rest of the CND and partially shocked. Such dark clouds are likely sites of pre-stellar cores (e.g., Sanhueza et al. 2012). In the context of research on star formation in the immediate vicinity $(\sim 2$ pc) of the supermassive black hole, these regions deserve further investigation.

\section{References}

Martín, S., et al. 2012, A\& $A$ 539, 25

Vasyunina, T., et al. 2012, ApJ 751, 105

Sanhueza, P., et al. 2012, ApJ 756, 60

Hirota, T., et al. 1998, ApJ 503, 717 
Shalabiea, O. M. \& Greenberg, J. M. 1994, A\&SA 290, 266

Watanabe, N. \& Kouchi, A. 2002, ApJ Lett. 571, L173

Yusef-Zadeh, F., et al. 2013, ApJ Lett. 764, L19

Brown, W. A. \& Bolina, A. S. 2007, MNRAS 374, 1006

Garrod, R. T., et al. 2007, A\&A 467, 1103 